Article

\title{
The Daily Spiritual Experience Scale: Overview and Results
}

\author{
Lynn G. Underwood
}

Research Integration, 37 Forest Drive, Chagrin Falls, OH 44022, USA;

E-Mail: lynnunderwood@researchintegration.org; Tel.: +1-440-708-8501

Received: 29 December 2010; in revised form: 5 January 2011 / Accepted: 10 January 2011 /

Published: 12 January 2011

\begin{abstract}
The Daily Spiritual Experience Scale (DSES) is a 16-item self-report measure designed to assess ordinary experiences of connection with the transcendent in daily life. It includes constructs such as awe, gratitude, mercy, sense of connection with the transcendent and compassionate love. It also includes measures of awareness of discernment/inspiration and a sense of deep inner peace. Originally developed for use in health studies, it has been increasingly used more widely in the social sciences, for program evaluation, and for examining changes in spiritual experiences over time. Also it has been used in counseling, addiction treatment settings, and religious organizations. It has been included in longitudinal health studies and in the U.S. General Social Survey which established random-sample population norms. It has publications on its psychometric validity in English, Spanish, French, Portuguese, German and Mandarin Chinese. Translations have been made into twenty languages including Hindi, Hebrew and Arabic and the scale has been effectively used in a variety of cultures. The 16-item scale does not have a psychometrically representative shorter form although a 6-item adaptation has been used. The DSES was developed using extensive qualitative testing in a variety of groups, which has helped its capacity to be useful in a variety of settings. It was constructed to reflect an overlapping circle model of spirituality/religiousness and contains items that are more specifically theistic in nature, as well as items to tap the spiritual experience of those who are not comfortable with theistic language. The scale has been used in over 70 published studies. This paper will provide an overview of the scale itself, describe why it has proved useful, and discuss some studies using the scale. See http://www.dsescale.org/ for more information on the scale.
\end{abstract}

Keywords: DSES; spiritual experiences; spirituality; religiousness; assessment; well-being; quality of life 
Abbreviations: DSES: Daily Spiritual Experience Scale; DSE: Daily Spiritual Experience; GSS: General Social Survey; BMMRS: Brief Multidimensional Measure of Religiousness/Spirituality; WHO: World Health Organization

\section{Introduction and Background}

The Daily Spiritual Experience Scale (DSES) is a sixteen-item self-report measure together with a brief introduction, designed to measure ordinary spiritual experiences (not dramatic mystical experiences such as near death experiences or hearing voices or seeing visions). It measures experiences of relationship with, and awareness of, the divine or transcendent and how beliefs and understandings form part of moment-to-moment features of life from a spiritual or religious perspective [1,2]. The DSES was constructed as a scale that allows for an "overlapping circles" concept of religiousness/spirituality. It was consciously constructed to have deep relevance for those comfortable with theistic religious language, but also to provide opportunities to respond for those who are not comfortable with a theistic view. The word 'spirituality' in the context of this scale refers to aspects of personal life that include the transcendent, divine or holy, "more than" what we can see or touch or hear. Details on intent of wording and of individual items and theoretical background can be found in two seminal papers on the scale [1,2]. Additional conceptual background can be found in other theoretical papers $[3,4]$.

As the author of the DSES, and one of the lead developers of the Brief Multidimensional Measure of Religiousness/Spirituality (BMMRS) [5], this author sees religiousness/spirituality as a multidimensional construct. The DSES makes a significant contribution to measurement of this construct and continues to be actively useful in studies. This paper is designed to: (1) Give a review of some of the background to the instrument's development; (2) give some of its psychometric properties; (3) discuss issues of adaptation, translation and interpretation; and (4) describe some areas of study in which it has proven useful including some empirical studies using the instrument.

This scale drew upon the author's pragmatic background in clinical medicine and epidemiology, where multiple ways to measure melanoma - a skin cancer-were tied to stage of diagnosis, which led to appropriately targeted efforts at education and improved early detection [6]. It also drew on the author's work with measuring stress and social support and, especially, on identifying how clarity in those measures and their interpretation was key to the effective development of various kinds of interventions [7,8]. It is important that empirical measures of religiousness/spirituality lead effectively to the ability to usefully develop attitudes, behaviors, practices and interventions that will lead to improvements for us as human beings. Religiousness/spirituality is not only a collection of variables to be manipulated to obtain a positive mental or physical health outcome, or improved quality of life. These variables can be considered as outcomes in their own right. And for many this is a more important consideration. As good scientists we are called to set aside our own preconceptions, gather information and interpret results, and present our results in ways that enable appropriate interpretation by others. We also need to realize that all empirical measures in this area approximate their goal. 
The DSES was designed to assess ordinary experiences of connection with the transcendent in daily life. It includes constructs such as awe, gratitude, mercy, sense of connection with the transcendent and compassionate love. It also includes items relating to awareness of discernment/inspiration and a sense of deep inner peace. It measures subjective experiences that form an integral part of daily life for many ordinary people. The goal of this instrument is to obtain a measure of various qualities of the spiritual life as it plays out in the experiential and emotional details of daily life. The experiences tapped by this instrument are feelings and sensations, rather than cognitive articulation of specific beliefs. For many people these experiences may have a highly charged emotional tone, for others, the sensations may seem less specifically emotional, and more like direct sensation or awareness. Some of the experiences that the DSES is attempting to capture can be best articulated using religious language; some do not require explicitly religious language. Some feelings or direct sensations are considered more important for particular religious traditions than others. Thus a breadth of particulars was included such as a sense of awe, a sense of thankfulness, feelings of compassionate love, mercy, and desire for divine closeness. It was not expected that everyone would have all of these experiences, but it was hoped that the spectrum would cover a variety of those present. One of the things that has helped the applicability of the scale has been this breadth and inclusiveness, while maintaining depth of meaning. Although the DSES can at times provide a useful proxy for spirituality, and operationalize one major aspect of spirituality, it does not capture the construct fully. Religiousness/spirituality is a multidimensional construct. Any complete operational definition of spirituality would need to consider the context of beliefs, practices, culture, and a variety of other factors, which can also include more negatively charged features of spirituality.

Details of the methods used in the development of the scale, conceptualization and design of each of the items and the Introduction, and results from the qualitative interviews, can be found in Underwood [1]. The development process included review of sources from theology, comparative religion, the social sciences, a review of available scales, and many in depth interviews with a large variety of people over time. Subsequently structured interviews were conducted to refine the items. These included a spectrum of religious perspectives and non-religious ones, gender, socioeconomic spread, educational levels, various countries and cultures accessed via a World Health Organization project and other contacts, in depth interviews with individuals for whom spirituality was central, and interviews with older children and adolescents as well as older adults. As the items were refined, feedback provided the ability to home in on the actual construct of interest, finding a group of words that would tap this for as many different kinds of people as possible, while still maintaining capacity to touch the depths of the construct.

In developing the scale there was an emphasis on maintaining an ability to answer the questions easily and directly without excess analysis of belief systems, or overly complex wording, even though those might be implicitly drawn on during item response. The initials DSES have also been used for a recent depression scale and so, more recently, it has been suggested that this scale be called Underwood's DSES for clarity (Table 1). 
Table 1. Daily Spiritual Experience Scale (with item numbers added). Introduction: "The list that follows includes items you may or may not experience. Please consider how often you directly have this experience, and try to disregard whether you feel you should or should not have these experiences. A number of items use the word 'God.' If this word is not a comfortable one for you, please substitute another word that calls to mind the divine or holy for you."

\begin{tabular}{|c|c|c|c|c|c|c|c|c|}
\hline & & $\begin{array}{l}\text { Many } \\
\text { times a } \\
\text { day }\end{array}$ & $\begin{array}{l}\text { Eve } \\
\text { day }\end{array}$ & & $\begin{array}{l}\text { Most } \\
\text { days }\end{array}$ & Some days & $\begin{array}{l}\text { Once in } \\
\text { a while }\end{array}$ & $\begin{array}{l}\text { Never or } \\
\text { almost } \\
\text { never }\end{array}$ \\
\hline $1^{*}$ & I feel God’s presence. & & & & & & & \\
\hline 2 & I experience a connection to all of life. & & & & & & & \\
\hline 3 & $\begin{array}{l}\text { During worship, or at other times when } \\
\text { connecting with God, I feel joy which } \\
\text { lifts me out of my daily concerns. }\end{array}$ & & & & & & & \\
\hline $4^{*}$ & $\begin{array}{l}\text { I find strength in my religion or } \\
\text { spirituality. }\end{array}$ & & & & & & & \\
\hline $5 *$ & $\begin{array}{l}\text { I find comfort in my religion or } \\
\text { spirituality. }\end{array}$ & & & & & & & \\
\hline $6^{*}$ & I feel deep inner peace or harmony. & & & & & & & \\
\hline 7 & $\begin{array}{l}\text { I ask for God's help in the midst of } \\
\text { daily activities. }\end{array}$ & & & & & & & \\
\hline 8 & $\begin{array}{l}\text { I feel guided by God in the midst of } \\
\text { daily activities. }\end{array}$ & & & & & & & \\
\hline $9 *$ & I feel God’s love for me directly. & & & & & & & \\
\hline $10 *$ & I feel God's love for me through others. & & & & & & & \\
\hline $11^{*}$ & $\begin{array}{l}\text { I am spiritually touched by the beauty } \\
\text { of creation. }\end{array}$ & & & & & & & \\
\hline 12 & I feel thankful for my blessings. & & & & & & & \\
\hline 13 & I feel a selfless caring for others. & & & & & & & \\
\hline 14 & $\begin{array}{l}\text { I accept others even when they do } \\
\text { things I think are wrong. }\end{array}$ & & & & & & & \\
\hline $15^{*}$ & $\begin{array}{l}\text { I desire to be closer to God or in union } \\
\text { with the divine }\end{array}$ & & & & & & & \\
\hline & & \multicolumn{2}{|c|}{ Not close } & \multicolumn{2}{|c|}{$\begin{array}{l}\text { Somewhat } \\
\text { close }\end{array}$} & Very close & \multicolumn{2}{|c|}{$\begin{array}{l}\text { As close as } \\
\text { possible }\end{array}$} \\
\hline 16 & In general, how close do you feel to God? & & & & & & & \\
\hline
\end{tabular}

(c) Lynn Underwood - contact author to register to use scale http://www.dsescale.org/ or lynnunderwood@researchintegration.org;

* signifies items that were used to form part of the BMMRS 6 item scale, DSE domain. 4 and 5 was combined: "I find strength and comfort in my religion." And 9 and 10 was also combined: "I feel God's love for me directly or through others.” These form part of the 6 item DSES referred to in the text. 


\section{Psychometrics, Adaptations, Translations and Scoring}

\subsection{General psychometrics}

Psychometric details are best found in various original publications cited here. Conceptual underpinnings and psychometrics are described in the original paper on the scale [2]. A list of the items with the introduction is found in Table 1. One must keep in mind that individual psychometric results depend on the assumptions made and the populations and datasets on which they are based. Chronbach's alpha's for the scale in English and in translation, for both the 16-item version and the adapted 6-item version (more later on why the 6-item version is not recommended), have been consistently high, 0.89 and above [2,9-11], for example 0.94 and 0.95 for the General Social Survey samples [12]. Item distribution and skew was assessed as adequate, showing a wide range of variability in responses [2]. Test-retest results have been reliable, with test-retest Pearson correlation of 0.85 over two days [2]. Bailly and Roussiau [4] and $\mathrm{Ng}$ et al. [11] also show good test-retest reliability in translations of the scale. Both versions were stable over time, internally consistent, and the forms were equivalent and valid in an all-African American sample [13].

Although there can be trait-like consistencies over time, the scale also has state-like characteristics, lending it to examinations of change over time, as variability can be documented. For example, it was used in a daily diary study of pain quite effectively [14], and in a study of effects of meditation on pain tolerance [15]. It has been used to measure change over time with various interventions in healthcare, substance abuse treatment, education, and religious settings further described in the Results section [16-18].

Each item in the scale was designed to tap a separate feature of spiritual experience, but it was hoped that there would be a cohesiveness of conceptual underpinning. The original paper on the scale's psychometrics demonstrated a single factor, with weaker loadings by certain items, especially the two compassionate love items (13 and 14, self-less caring and accepting others) [2]. These two items often have a weaker loading, but conceptually are important to the breadth of content addressed in the scale.

Translations of the scale into Chinese, Spanish, French, Brazilian Portuguese and German have shown loading on a single factor. The Chinese translation showed a Chronbach's alpha of 0.97 using the single factor model that emerged through Principal Component Analysis [11]. The Spanish translation tested in the Mexican population had a Chronbach's alpha of 0.91, with a single factor solution, with items 2, 13 and 14 loading less strongly [10]. The German translation of the 16-item scale has shown a single factor structure, and supports the unidimensional structure of the scale and provides other psychometric support for its use in that population [19]. Confirmatory Factor Analysis shows a single factor in a European French population [9]. Kalkstein and Tower [20] show a single factor, even though there was weaker loading on the two compassionate love items (13 and 14). Robinson et al. 2007 found a one factor solution in an alcoholic population in treatment, with a 0.92 Chronbach's alpha [17].

Ellison and Fan, using the 2004 General Social Survey (GSS) results, examine both a one-dimensional and two-dimensional approach [12]. They divided the scale into items containing eight items specifically mentioning 'God' and a separate group, they called 'Non-Theistic' including 
the remaining eight 'non-God' items, and did the same for the 6-item scale in the 1998 GSS. They provide some support for a global single-factor model, while also supporting a two-factor model. A California sample of a substance abuse population showed a two factor structure [21]. The division was different than Ellison and Fan, and they called their item subgroups theistic $(1,3,4,5,7,8,9,10,15)$ and self-transcendence $(2,6,11,13,14)$, after eliminating items 12 (thankful for blessings) and 16 (close to God). It is expected that for some, the more explicitly theistic items will be responded to differently, but this does not necessarily make it a separate factor in the scale as applied more generically. The single factor solution is the dominant one at present.

Labels assigned to subgroups can unduly influence interpretations of results. Some items which might fall into the theistic grouping on face value might be able to be responded to positively by a non-theist. An example of this might be: "I feel God's love for me through others." Given the introductory sentences for the scale ("A number of items use the word God. If this word is not a comfortable one for you, please substitute another idea which calls to mind the divine or holy for you.") this item has been reported with high frequency by non-theists, as discovered in the initial qualitative developmental research on the scale. Another example of an item that cannot be clearly given a theist or non-theist distinction is: "I desire to be closer to God, or in union with the divine." From the original development interviews, this item for example reflected spiritual experiences of an agnostic scientist, who would not be classified as a theist yet felt this item captured experience. "I feel a connection to all of life” is particularly salient for non-theists, however as environmental awareness increases, this may become increasingly relevant for theists. For example, connection with the natural world is strongly emphasized in the Franciscan Christian spiritual tradition. Although divisions of the scale on these lines may prove useful for some populations, the scale was designed based on research that demonstrated that these divisions are not as clear cut as we often assume. It is important not to create boundaries or categories that may not exist in the mind of the respondent. It is expected that cultures will also differ as to if, or how, this kind of division might be useful.

An analysis of the entire set of long form items included in the NIA/Fetzer Multidimensional Measurement booklet, included the 16-item DSES, and found that the DSES items form a useful and psychometrically valid single factor in that larger group of items [22]. Idler et al. found that the six DSE items constituted a single dimension when the entire BMMRS on the GSS was analyzed [5].

Continuing to consider the scale as uni-dimensional seems warranted at this time, taking these various factor analysis investigations into account. Since each item was designed to tap a dimension of spiritual experience, it is expected that items will perform differentially in various studies. The inclusion of the Introduction is important to enable effective factor analysis of the scale to take place. Premature separation of items into subgroups can actually get in the way of understanding.

\subsection{Versions, adaptations, translations}

It is tempting to tweak a scale. One reason is our own biases. We read an item and it does not seem to fit how we and those we know personally describe things, and then we make what seems like a sensible change. Our biases in the religious/spiritual realm can be quite strong and we can often be unaware of them. A few authors have changed the wording of the scale items substantively and still call their versions a scale of Daily Spiritual Experiences. This is strongly not advised. The wording of 
this scale is critical to its effectiveness and ability to tap the construct of interest. Changes in wording can strongly affect performance, as well as the capacity to compare with the many studies that have used the scale. If the wording is changed, the capacity to use the available norms is adversely affected, as is the ability to compare results between studies. The DSES does not meet all needs, and other scales should be used to address other needs.

The word "God" was consciously used in the DSES. For more detailed rationale see Underwood [1]. A recent study of 8-12 year-olds in Canada substituted "higher power" for God in the God items [23]. Although the study did show predictive value in the DSES items used, interviews by Underwood while developing the scale showed a lack of clarity with the phrase "higher power," and this substitution is not recommended. Not all view God as either higher or a power. Some view God as a collaborator in life's decisions [24], or someone who "has your back" [25]. 'God' may be a contentious word for many, but the Introduction to the scale enables people to make their own substitutions as needed rather than the investigator deciding to edit it for them, perhaps erroneously. The scale's Introduction states: "A number of items use the word God. If this word is not a comfortable one for you, please substitute another idea which calls to mind the divine or holy for you." Additionally, in the scale there are many non-God items that provide additional scope for responses. The DSES affirms the value of not watering down the concept of spirituality by discarding religious terms, but leaving space for spirituality that is religiously grounded and framed, as well as that which may not be.

Response categories for the scale are also important. The scale is a frequency scale for a reason. It encourages users to search their memory for specific experiences and attempt to measure frequency. A study using Rasch analysis and item response theory on the Portuguese version in Brazil found that overall the categories were appropriate and discriminating [26].

Sometimes adaptation to a particular population merits a specific change. Examples of appropriate adaptations are rare but include substituting "Allah" for "God" in the items for a study of Afghan refugees [27], and substituting "Creator” for "God” for use in a study of Zuni Pueblos an indigenous American population in an ongoing study by Kamilla Venner at the University of New Mexico. Because of the homogeneity of these groups and early testing of these forms to identify equilibration with the original concepts, the adaptation was appropriate in these cases. The scale has been republished in a number of venues, and sometimes the wording is not accurate. Sometimes tables used in various publications are inaccurate regarding item wording. It is important to refer to the Underwood peer-reviewed publications in this regard.

\subsubsection{Using selected items and subsets}

Various researchers have used subsets of the 16-item scale for particular purposes. This can be appropriate, as long as the rationale is sound. Two of the items, for example, ("I feel a selfless caring for others" and the mercy item, "I accept others even when they do things I think are wrong”) have been used as a compassionate love subscale in a number of studies [28,29].

Each individual item in the DSES taps one particular aspect of ordinary spiritual experience and various items have been used alone in studies. The "I feel deep inner peace" item may be effective when used alone at end of life. With its response categories of "many times a day" to "never or almost 
never” and its particular language, it taps a spiritual sense rather than an absence of anxiety, or merely reaching resolution of interpersonal conflict. Researchers have also found "I am spiritually touched by the beauty of creation” a particularly useful item. "I feel thankful for my blessings" could be used alone if one was particularly seeking an individual item for the spiritual experience of the construct of felt spiritual gratitude. Recent studies have focused on the utility of daily acknowledging gratitude and this item is useful in this regard [30]. Individual items may prove helpful in indicating interventions that may be appropriate in various circumstances.

There are different opinions on numbers of items necessary to include in scales. Epidemiologists are continually trying to slim down numbers to reduce burden on subjects. Some psychometricians believe that multiple items are needed to make up any single factor. However others use single items frequently and they can be quite predictive [31]. Each item in the DSES was designed to tap a particular aspect of spiritual experience, and each may ultimately prove significant in its own right depending on the particular need of the research or evaluative use. At the analysis stage individual items may prove useful even if the entire scale is administered, as long as statistical integrity is maintained, for example in hypothesis generation and theoretical rationale. It is important to keep in mind that including the diverse set of 16 items is valuable, as the spectrum of experience is more fully covered.

Including the Introduction is important for the scale. The introductory comments are designed to create a relaxed environment, to instill a sense of respect for the respondents' unique experiences, and to lower the social desirability responses, as well as including language that enables people to make personal adaptations when necessary.

\subsubsection{The 6-item form of the scale}

Currently there is no psychometrically representative shorter version of the DSES. The full 16-item scale is the recommended version of this scale. However, following the finalizing of the 16-item scale, a 6-item adapted DSE scale was developed to be used in the Brief Multidimensional Measure for Religiousness and Spirituality (BMMRS) [5]. The six items are not ideal in many ways, as the wording is not identical and does not fully represent the breadth and depth of the construct. The double-barreled nature of two of the items, and the alternative wording of the strength and comfort item (using just "religion" rather than "religion or spirituality"), are not the best choice for use. See Table 1 for items included in the 6-item version. It was developed ad hoc as the entire BMMRS as a whole was, to meet the need of getting a brief measure onto the GSS at that time, in order that a multidimensional measure could be utilized in a variety of studies with immediate needs. The BMMRS includes items on religious history, affiliation, public practice and religious coping. Given the ad hoc nature of all the subscales, it is advised that researchers consult the original authors of each subscale to get up to date recommendations for the appropriate items to use to tap the construct of interest as they design new studies. Some minor alterations to the 6-item DSE scale have included adding "spirituality" to the strength and comfort item to produce more agreement with the original items. Despite the limitations of the 6-item scale, it has been found to be highly correlated with the longer version with similar effect sizes on selected variables present in the GSS [12], and may be appropriate for studies where item number limitation is critical. Studies incorporating the 32- or 38-item BMMRS frequently find that the 
DSE subset of six items is one of its most predictive elements. This has encouraged various researchers to use the full 16-item DSES as their research moves forward.

\subsubsection{Other shorter forms}

Dropping individual items to produce a shorter form may be a possibility in the future, but considerations in various cultures indicate maintaining all 16 items at this stage. The strength and comfort items show very high inter-item correlation in some cultures; however it is currently recommended that both be maintained, following interviews showing distinct qualities for each, and results in testing in translation that showed greater separation. Even during the original development of the scale, it was tempting to remove the last item ("how close do you feel to God?") that is scored differently. It was originally included to allow calibration of the item: "I desire to be closer to God or in union with the divine." Further work may enable appropriate ways to develop a shorter psychometrically valid form if needed, by dropping some items while continuing to maintain a balance of items.

\subsubsection{Translations}

The DSES has particular qualities that lend it well to cross-cultural research and use in a variety of religious and cultural settings. Translation needs to be done carefully, as language is particularly important and cultural sensitivity is needed. A stringent methodology of back-translation is encouraged, and some involvement of those from a variety of perspectives, and with understanding of the potential depth of the constructs, is required to enable the translators to capture the intent of the items fully. Involving the scale's author in translations can be quite helpful, particularly when making choices between particular options. Unfortunately literal translation efforts do not capture the underlying constructs. Words and phrases need subtle translation in context to produce accurate translations of, for example "close,” “joy,” "connection to all of life," "feel guided," "presence," “divine,” holy,” “accept others” and "self-less caring.” Underwood [1] includes elaboration of intent of particular items, very useful in translation development. An example of issues that need careful attention is the selfless caring for others item. Tapping the construct of other-centered love may require different phrases in different languages. The guided by God item is designed to provide opportunity to respond concerning a sense of nudges by the divine, more subtle inner guidance as well as more clear guidance. The accept others item is getting at deep respect for the other person, a merciful attitude. Translation needs to give adequate attention to these subtleties such that the complexity and variations of the construct continue to be tapped in the new language and cultural setting. Also testing in a variety of populations can assure that the wording is fully understood by a variety of people.

The psychometric properties of the DSES have been demonstrated in Spanish [10], French [9], Mandarin Chinese [11], Brazilian Portuguese [26], and German [19]. Other translations in use include: Arabic (Saudi Arabia), Egyptian, Greek, Hebrew, Hindi, Hungarian, Italian, Korean, Lithuanian, Nigerian, Pakistani, Persian, Polish, Portuguese (Portugal), and Vietnamese. 


\subsection{Scoring and analysis}

Details on scoring and analysis are found in the Underwood [1] paper and Underwood and Teresi [2]. Often more frequent daily spiritual experience is represented by lower crude totals or mean scores. An algorithm is used to create a more intuitively understood higher score for more experiences, which is helpful in describing correlations. Using "higher DSE's" rather than "DSES score" is a way around this problem in the text, and using the transformed scores in tables can also help here. Occasionally the term "higher DSES" is incorrectly used to describe more spiritual experiences, just assuming the transform. This can be somewhat confusing, so it is important to read the texts clearly.

Mean scores are usually the most useful for analysis, rather than total score. This also allows for examination of individual items or subgroups. Individual items that bear theoretical connection to the outcome of interest might $a$ priori be hypothesized to have a connection in a particular study and could be examined in this way.

Another way of doing analysis is to dichotomize the responses. Where significant break points can best be drawn may differ from one study to another. It is possible that grouping "many times a day" and "every day" could provide a significant break point that has implications. As well, dividing into never or almost never, and all other frequencies of experience, may also yield significant results, depending on the research question of interest.

\section{Results Using the DSES}

There are over 70 published studies using the DSES to date. Most use the full 16-item scale; however some use the 6-item DSE set of items, and a few use smaller subsets of items. The following summary is intended to give an idea of the kinds of uses the scale has been put to and some results. The original papers, those described here and others can be read in depth to provide more details. Although there is no charge for using the scale, permission from and registration with Underwood is required for scale use and translation, in order to track its use and connect researchers with one another and with current information, e.g. translation data, and ongoing studies in fields of interest.

\subsection{Demographics}

The DSES has been included in the General Social Survey (GSS). This allows for examination in relationship to a variety of other variables in this random sample public domain database [32,12]. Underwood [1] gives individual item breakdown of DSES item responses from this survey. For example, for “I feel God's presence," 37\% experience this many times a day to most days, with $22.4 \%$ never or almost never experiencing this. It is more common in the general U.S. population to feel God's love through others (40.7\% many times a day to most days) than directly (35\%), with $20.8 \%$ reporting never feeling God's love directly, and 15.0\% reporting never feeling God's love though others. In response to "I am spiritually touched by the beauty of creation," $25.7 \%$ reported this many times a day to most days, and $20.3 \%$ never or almost never. One of the least frequently reported items was "I feel thankful for my blessings," with only $11.2 \%$ of the population reporting this many times a day to most days and $30.9 \%$ not at all. $17 \%$ express that they feel as close as possible to the divine, and 
$10.2 \%$ report feeling not at all close. This open-access dataset also provides the opportunity in the future for comparison with data from other countries.

The emerging data from studies of the DSES in translation provide opportunities to compare inter-culturally. For example individuals from Mexico show more frequent DSE's than in U.S. general population samples [10]. A study comparing responses from Mexico and Basque Europeans found some significant differences in responses on the DSES, and more DSE was correlated with better well-being and other variables in both populations [33]. Self-identified atheists in the Basque sample in this study reported quite high DSE's. It is important when doing cultural comparisons to eliminate confounding variables when possible. The DSES has been placed on a longitudinal study of African American health, and it has been validated in that population [34]. African Americans generally report higher DSE's. It has also been shown to be useful in various ongoing studies of Hispanic Americans, Hawaian Americans and Native Americans.

Women generally report higher DSE's than men in U.S. samples; however as studies using the translations of the scale emerge, the U.S. appears to be the exception regarding differences between men and women regarding DSES scores. The study of the DSES in Hong Kong showed no gender difference as well as no differences by age or educational level [11]. A study of European Basques and Mexicans showed no gender differences in either group [33]. The study of the DSES in a French population showed no gender differences in distribution of responses [9]. There is an emotional quality to a number of the items. It may be more acceptable for men to report emotions in some cultures than others. It is worth continuing to explore whether this also encourages males to be open to spiritual experiences more in some cultures than in others. It also is possible that some cultural differences relate to the predominant religious influences in a culture, regardless of affiliation.

There also may be some different ways in which DSES interacts with other parts of life for males and females. In one study of U.S. male college students, higher levels of DSE correlated with valuing influence and service in their career choices, whereas for females no significant relations existed among these constructs [35]. The GSS gender data showed differential outcomes for men and women regarding the effects of various religious variables and well being and health, finding that for women, higher DSE's were more predictive than religious attendance of well-being and health [36].

\subsection{DSES and physical health-related outcomes}

In a cross-sectional survey of more than 800 consecutively admitted patients at Duke University Medical Center, Koenig and colleagues found higher DSE's linked to fewer days of long-term care required [37]. They also found that spiritual experiences, prevalent in older hospitalized patients, are associated with greater social support, better psychological health, and better physical health [38].

In a longitudinal community-based study of 6,534 older adults, DSE's measured using five of the more explicitly theistic items were associated with better self-reported health [39]. Positive associations of DSE's with health were independent of public religious activity in the GSS [36]. Kalkstein and Tower [20] also showed positive correlation of DSE's with self-rated health.

DSE's, physical activity and healthy diet all contributed to higher physical well-being in a stress deterrent model, as counterbalancing stress in a study of healthy lifestyle behaviors in adults [40]. In arthritic older adults, DSE's were more common in those with arthritis, but not with other chronic 
diseases such as high blood pressure [41]. In a few studies no links between DSE's and specific health outcomes were found. This is not surprising. For some conditions, DSE's might form a part of dealing with the challenges of daily life, while not exerting a major influence overall on progression of or development of disease across the board. DSE's may weigh in on both sides of the equation for a variety of disease processes.

At least three studies of pain have used the DSES. A daily diary study showed DSES changes over time in rheumatoid arthritis patients, where frequent daily spiritual experiences linked with higher levels of positive mood, lower levels of daily negative mood, and higher levels of each of the social support domains [14]. It was not linked to experience of pain; however attention to pain has also been connected with diminished suffering [42]. Rippentrop [43], in 157 pain patients showed that DSE's predicted mental health status but not pain outcomes, and suggested that pain encourages engagement with DSE's. Wachholtz and Pargament used the DSES in a study of pain outcomes, where spiritual meditation groups, with higher post-intervention DSE scores, had increased pain tolerance [15].

Examining all the BMMRS subscales in a study of 168 assorted medical patients, it was found that only the DSES items significantly predicted SF-36 General Health Perception, after taking into account demographic and other health status variables. DSE items accounted for an additional 6\% of the variance [44].

In a study of 157 Congestive Heart Failure patients, DSE's were not linked with depression; however they were linked with increased self-efficacy in dealing with one's illness [45]. Since the DSES has been included in a number of ongoing longitudinal databases there is future opportunity for secondary data analysis over time, not just with the health outcomes, but also with the other variables embedded in those databases. It has been included in various on-going health studies that often do not include spiritual or religious variables for a variety of reasons.

\subsection{DSES, work-related outcomes, and burnout}

The Chinese translation was used in a study of 245 professional and support staff in a rehabilitation services complex in Hong Kong, where DSE's were negatively correlated with anxiety, depression and total burnout [11].

A study of secondary school teachers in Nigeria showed that higher DSE was independently correlated with less work family conflict [46]. DSE's in teachers in England were correlated with having increased inner resources and finding deeper meaning in their work [47].

Daily spiritual experiences mitigated physical, cognitive, and emotional forms of burnout for medical and mental health practitioners working in palliative care [48]. Physical fatigue, cognitive weariness and emotional exhaustion were the elements of burnout assessed in this study of professionals.

In a study of correlations with being employed in a public service occupation, the combined DSES item, "I feel God's love directly or through others," was a predictor of working in public service compared with other occupations, even after controlling for religion, whereas a non-DSES general spirituality item was not [49]. High DSE's strongly predicted attitude towards and utilization of religion in social work practice, whereas organized religion measures were not predictive [50]. A study of Protestant ministers in Germany showed more DSE’s correlated with diminished burnout [51]. 


\subsection{DSES and relationship outcomes}

In a study of how spouses help one another following trauma, partners who reported more emotional regulation and higher levels of daily spiritual experience provided more help to the victim of trauma. Although emotional regulation was initially a predictor, once other variables were controlled for, only DSES remained a significant predictor [52]. Examining the entire set of BMMRS dimensions (e.g., meaning, private practices, affiliation, religious coping), and reported marital happiness on the GSS, Bell found that of the BMMRS dimensions, a married man's daily spiritual experiences was the domain most strongly correlated with his self-reported marital happiness, followed by his organizational religiousness, and beliefs concerning forgiveness. Forgiveness was most strongly correlated with a married woman's self-reported marital happiness, followed by her daily spiritual experiences [53].

Brelsford et al. found that spiritual disclosure in 378 adults correlated with DSE's, which was also correlated with measures of generativity [54]. The two compassionate love items on the full DSES have been used in a number of studies [28,55]. They have been useful in studies of altruism and self-giving behaviors and attitudes, particularly when motivation is of interest. Lower levels of daily spiritual experiences correlated with having fewer close friends among a mixed sample of 409 adults and 84 older adults [20]. In a longitudinal study of older adults, positive association was found between social network integration and spiritual experiences in older adults [39].

In 85 individuals grieving the death of a significant person in their lives, those evidencing high DSE's showed lower levels of problematic grief affect. Church attendance influenced affect only to the extent that it was positively correlated with DSE's [56].

\subsection{DSES in adolescents}

Adolescents are often neglected in religiousness/spirituality research. The DSES was originally developed by including interviews with adolescents. The scale has been validated in adolescents, where in an ethnically diverse sample of 12-18 year olds in New York City it has shown good internal consistency and good test-retest performance [57].

Van Dyke et al. found more frequent DSE's to be a significant predictor of higher psychological adjustment and lower psychological distress in a group of urban lower socioeconomic status adolescents [58]. They also found in this group that daily spiritual experiences are associated with positive affect for males and females, and a predictor of life satisfaction for adolescent males. A regression analysis of 615 adolescents showed that daily spiritual experiences, forgiveness and positive religious coping were associated with less depressive symptomatology exclusively in girls [59].

Park et al. studied 167 young adults following diagnosis with a variety of cancers to better understand what motivates the kinds of self care health behaviors particularly required in these young people. Results showed that religious attendance had little impact on health behaviors, while daily spiritual experiences were related to greater performance of health behaviors [60]. Self-assurance only partially mediated these effects. 


\subsection{DSES and addictions}

Robinson et al. followed 123 alcoholics for six months following entry into treatment. Analysis showed that increases in DSE's (but not positive religious coping or forgiveness) were associated with increased odds of no heavy drinking at six months, even controlling for AA involvement [17].

In an analysis of 198 alcoholics using two subsets of DSES items, both subsets were correlated with longer sobriety and helping behaviors [21]. Lower alcohol intake has been associated with higher DSE's [2]. The DSES currently forms part of an ongoing study of adolescent alcohol abuse and helping behaviors. Shorkey and Uebel reviewed 10 measures for use in addiction research, and concluded regarding the DSES that "the usefulness of this scale for assessing the spiritual experience of a person in treatment and recovery may be profound" [61].

\subsection{DSES and psychological measures}

The GSS data shows a robust positive association between DSES and happiness, excitement with life, self-esteem, and optimism. By contrast, DSES demonstrated little relationship to negative affect in this dataset. DSES effects are present no matter what the gender, age, socioeconomic status, race [12].

Bailly and Roussiau, using a DSES translation in 165 French adults age 65 and older, showed higher scores associated with higher life satisfaction and self-reported good health, but no relationship with depressive symptoms [9].

In a study of male prison inmates, increased DSE was linked to better emotional health [62]. Ciarrochi and Deneke showed relationships with positive emotion and cognitive well-being above and beyond predictions from personality variables, public religious factors, gender and age [63,64]. Data from a variety of sources showed DSE was negatively associated with state-trait anxiety, and perceived stress and positively correlated with optimism, perceived social support and quality of life [2]. Moderate links have been shown between higher DSE's and greater well-being in widows and widowers [65]. In inner city elders, having better mental health was correlated with higher DSE [66].

In groups of European Basques and Mexicans, Mayoral et al. found that DSE was positively correlated with life satisfaction, positive affect, and psychological well-being, and negatively correlated with indicators of anxiety and depression. They found no correlations with the Big Five personality factors [33].

DSE's are not uniformly associated with depression across the board. The scale was designed such that one could respond positively to the items even when depressed or experiencing negative affect. However, an inverse relationship of DSE's with depression was found in some groups. In adolescents DSE predicted less depression [59]. The DSES was put on a National Institutes of Health longitudinal study of post heart attack outcomes. No link was found to survival or re-infarction, however higher DSE correlated with less depression [67]. In a group of arthritis patients, higher DSE was correlated with more energy and less depression [41]. Mofidi et al. found a relationship of more DSE's to less depression in 630 adults, an association moderated by age and stress [68]. Suggested mediators were optimism, volunteering and social support. In a cross-sectional survey of more than 800 consecutively admitted patients at Duke University Medical Center, Koenig and colleagues found significant positive associations between the DSES and social support and levels of cooperativeness, and inverse 
associations between the DSES and depressive symptoms [37]. Skarupski et al. found an inverse association between frequency of spiritual experiences and depressive symptoms in older adults [39].

In 497 adults, lower levels of spiritual experience were associated with higher levels of depression, anxiety, loneliness, and fewer close friendships, controlling for other religion, age, gender and education [20]. In survivors of domestic violence, higher DSES predicted fewer depressive symptoms and higher levels of social support [69]. The positive relationship between loneliness and depression, significantly associated among homebound older adults, was weaker among the older persons who reported higher scores levels of DSE [70].

\subsection{DSES as a measure of spiritual outcomes and change over time}

When people have been interested in examining change in spirituality over time, they have often used the DSES. It has been used to measure change following various interventions in healthcare, substance abuse treatment, education, and religious settings [16-18].

A control group study of the effects of two 1-day spiritual retreats on nurses' spirituality in a group of 199 critical care nurses, showed higher DSE's at the end of the retreat and six months post retreat [71]. In another study, the investigation of the efficacy of a self study program designed to teach nurses how to talk with patients about spirituality also showed changes in DSES scores using pre and post-test evaluations. In comparing nurses in the field with student nurses, the DSE scores of the students increased more than the practicing nurses over the course of the intervention [16].

A study looking at the effects of face-to-face prayer for patients with depression and anxiety showed that DSES scores in patients remained higher a month following the intervention [72]. A Canadian holistic medicine outcomes database has listed the DSES as one possible outcome measure [73].

A controlled trial of spiritual direction in a substance abuse population did not show an effect of the intervention on substance intake, as both groups improved over time. However the DSE's increased somewhat over the course of the intervention, and remained so six months later, whereas psychological variables showed less improvement over the course of the intervention compared with treatment as usual [74]. An online program intended to cultivate sacred moments used the DSES as the outcome measure for the 83 participants [18]. The control group wrote daily about their lives. Both groups showed decreases in perceived stress and increases in DSES over time and at six week follow-up.

The DSES is often used when others are developing scales of religious/spiritual variables to be used in specific contexts. For example, Cole et al. developed a spiritual transformation scale to be used in cancer patients, and found their scale highly correlated with the DSES [75]. Spiritual growth correlated positively with DSES and spiritual decline varied inversely. Hall, when developing a scale of spiritual maturity, in the Episcopal faith tradition, found higher scores on certain DSES items in "well-formed" Episcopalians [76].

Wachholtz and Pargament used the DSES as an outcome measure in their study of secular versus spiritual meditation. Spiritual experiences increased over time, more so in the spiritual than secular meditation group, although both groups showed some increase in DSE's [15]. 


\section{Conclusions}

With a respect for theistic traditions built into it, as well as inclusion of items that are often more widely accessible, the DSES has the capacity to both bridge differences and find common ground. The experiences addressed have importance to many people and can provide a connection to other aspects of life. As religious connections becomes stronger for many, and at the same time many people become alienated from organized religion, this instrument can address the religiousness/spirituality of a variety of kinds of people. The focus on experience rather than belief helps this, and its intercultural utility has been confirmed in a variety of cultures world-wide. Differences and similarities may influence various features of life.

Many report these experiences frequently, both those who are explicitly religious and those who are not, showing that relationship with the transcendent is a reality of life for many people on a regular basis. And this relationship has a connection with other parts of life, as found in various studies. For some it is an intimate relationship with a divine person, for others with a divine being beyond personhood. For these many people, this is a genuine relationship. For others, the connection is with a transcendent in a more diffuse sense, but particularized in various moments. The DSES has a capacity to tap into a sense of the real beyond what is seen or touched, a sense that exists for many people across religions and cultures. Formulation in a numerical scale allows statistical analysis and comparative work (see Table 1).

The full DSES complements other scales and can be usefully combined with scales of attendance, affiliation, coping, and private and public practice to flesh out a larger construct of religiousness/spirituality. The DSES is more acceptable than many scales to non-religious researchers and respondents partly due to the substantive section of non-explicitly religious questions. On the other hand, many religiously oriented researchers and respondents are reassured to see that orientation also reflected. It does not require exclusive separation of the constructs. One of the strengths of using social science tools is that they can help us to better understand those who are not like ourselves. The utility of this instrument is linked to its development. Using a theoretical framework and extensive qualitative testing together with a strong qualitative verification phase helped link the measure to the constructs of interest. Many translations of the scale exist and are proving useful in a variety of cultures worldwide confirming its wider applicability. U.S. population data norms for the scale exist. It has a solid psychometric base. Links have been established between the DSES and a variety of other variables. The concreteness of the items facilitate recall and accurate self-report.

Our relationships with others can be informed by the studies using the DSES in significant ways. The scale is relational in construction, and it is not surprising that scores on the scale have correlations with our relationships with others in concrete ways. The compassionate love items describe moments when people stretch out to those around them in care and acceptance and the two love perception items describe moments that perceived care flows in from a transcendent source either directly or through individuals. Although beliefs that "God loves us," or that one ought to love others are both important, the DSES measures a felt sense of this love as it touches daily life, and might affect our decisions, attitudes and actions. The DSES provides the opportunity to examine how transcendent love and care may help to fuel love and care for others [77]. 
Studies in adolescents show particular promise in use of the instrument. Less adherence to organized religion is being found in adolescents, and it may be possible to assess how spirituality may still be relevant using a tool such as the DSES. Older adults are another group of special interest. The aging population is facing challenges and the proportion of elders is growing. The increasing role spirituality/religiousness may play in life as we age, seen using the DSES and other measures, may provide opportunity for increased utilization of a significant resource for older adults. Studies in addiction have shown the role of spirituality in dealing with the damages of addiction, and the DSES is a sensitive tool to capture some features of spirituality that might protect from substance abuse. Results showing the protective role of DSE's in burnout also point to additional resources in difficult times for many others.

As an outcome measure it has provided an opportunity to assess changes over time using a spiritually sensitive instrument. It is currently being used as an assessment tool in ministry programs in religious settings, as well as assessing effects of training programs for health and social service professionals. It can be examined both as a factor correlated with others and involved in a causal chain, and also as a measure of certain spiritual outcomes. Opportunities to study DSES scores over time can continue to yield more information. Do practices that increase spiritual experience have positive effects on a variety of other outcomes? Which practices are most likely to increase spiritual experiences? Are some spiritual experiences particularly sensitive to certain kinds of interventions? Religious practices from faith traditions tested over time provide a rich resource here, as well as possibly more non-traditional approaches [3].

Although it was originally developed for use in health studies, the DSES continues to be used even more in other areas as described here. Ongoing studies are taking place: In both Pakistan and India examining spirituality in the workplace; Saudi Arabia in psychiatric work; the Philippines in health care training; on post-traumatic growth in Italy; Ireland in a study of the effects of guided imagery in prayer on spiritual growth; in humanitarian workers in Malaysia; in a study of adolescent offenders; in Puerto Rico examining psychosocial outcomes; with doctoral students in clinical psychology, social work, religious studies, exercise science, ministry and occupational therapy. It is being used in the field in churches, pastoral care, health settings, palliative care, addiction treatment, and social work. The spectrum of translations noted in this paper indicates studies being conducted in various cultures worldwide.

What are the implications for action and attitude given the growing body of results from the use of the DSES? There is growing indication that if one can enhance spiritual experiences, this, in turn may in various ways enhance the lives of the persons having them. The positive life contributions of social support, and negative contributions of loneliness, continue to be supported by research. The DSES measures "relationship with the transcendent" which is not dependent on social circumstances and may provide resiliency for many. But also, and possibly more importantly, from a religious/spiritual perspective DSE's in themselves are of value. They enrich the spiritual lives of those who value relationship with the transcendent as something of significance and importance in and of itself. However they also seem to be linked to a variety of positive outcomes and psychological states and good workplace and relationship outcomes. This is worth attending to when we consider the additional value spiritual and religious experiences may bring to people's lives, and to those around them.

Research using the DSES has the potential to continue to inform us about how ordinary spiritual experience and mental and physical health and well-being interact with one another. It also continues 
to inform us about the nature of spiritual experiences, how they may form a part of life for many kinds of people, and gives insight into various aspects of spiritual/religious life and its development.

\section{References and Notes}

1. Underwood, L.G. Ordinary spiritual experience: Qualitative research, interpretive guidelines, and population distribution for the Daily Spiritual Experience Scale. Arch. Psychol. Rel. 2006, 28, 181-218.

2. Underwood, L.G.; Teresi, J.A. The Daily Spiritual Experience Scale: Development, theoretical description, reliability, exploratory factor analysis, and preliminary construct validity using health-related data. Ann. Behav. Med. 2002, 24, 22-33.

3. Underwood, L.G. A working model of health: Spirituality and religiousness as resources: Applications to persons with disability. J. Relig. Disabil. Health 1999, 3, 51-71.

4. Underwood, L.G. Now bounded, now immeasurable: Perspectives on time in disability, in suffering and at end of life. Med. Humanit. 2007, 33, 11-15.

5. Idler, E.; Musick, M.; Ellison, C.; George, L.; Krause, N.; Ory, M.G.; Pargament, K.I.; Powell, L.H.; Underwood, L.G.; Williams, D.R. Measuring multiple dimensions of religion and spirituality for health research. Res. Aging 2003, 25, 327-364.

6. Underwood, L.G.; Lowry, W.S. The incidence and pathogenesis of invasive cutaneous malignant melanoma in Northern Ireland. Br. J. Cancer 1986, 53, 75-80.

7. Cohen, S., Kessler, R.C., Underwood, L.G. Measuring Stress: A Guide for Health and Social Scientists; Oxford University Press: New York, NY, USA, 1997.

8. Cohen, S., Underwood, L.G., Gottlieb, B.H., Social Support Measurement and Intervention: A Guide for Health and Social Scientists; Oxford University Press: New York, NY, USA, 2000.

9. Bailly, N.; Roussiau, N. The Daily Spiritual Experience Scale (DSES): Validation of the short form in an elderly French population. Can. J. Aging 2010, 29, 223-231.

10. Mayoral Sanchez, E.; Underwood, L.G.; Laca Arocena, F.; Mejía Ceballos, J.C. Validation of the Spanish version of Underwood's Daily Spiritual Experience Scale in Mexico. Int. J. Hisp. Psychol. 2011, in press.

11. Ng, S.-M.; Ted, C.T.; Fong, E.; Tsui, Y.L.; Friendly, A.-Y.; Law, S.K.W. Validation of the Chinese version of Underwood's Daily Spiritual Experience Scale-transcending cultural boundaries? Int. J. Behav. Med. 2009, 16, 91-97.

12. Ellison, C.G.; Fan, D. Daily spiritual experiences and psychological well-being among US adults. Soc. Indic. Res. 2008, 88, 247-271.

13. Loustalot, F.; Wyatt, S.; Boss, B. Psychometric examination of the Daily Spiritual Experiences Scale. J. Cult. Divers. 2006, 13, 162-167.

14. Keefe, F.J.; Affleck, G.; Lefebvre, J.; Underwood, L.; Caldwell, D.S.; Drew, J.; Egert, J.; Gibson, J.; Pargament, K. Living with rheumatoid arthritis: The role of daily spirituality and daily religious and spiritual coping. J. Pain 2001, 2, 101-110.

15. Wachholtz, A.B.; Pargament, K.I. Is spirituality a critical ingredient of meditation? Comparing the effects of spiritual meditation, secular meditation, and relaxation on spiritual, psychological, cardiac, and pain outcomes. J. Behav. Med. 2005, 28, 369-384. 
16. Taylor, E.J.; Mamier, I.; Bahjri, K.; Anton, T.; Petersen, F. Efficacy of a self-study programme to teach spiritual care. J. Clin. Nurs. 2009, 18, 1131-1140.

17. Robinson, E.; Cranford, J.; Webb, J.; Brower, K. Six-month changes in spirituality, religiousness, and heavy drinking in a treatment-seeking sample. J. Stud. Alcohol Drugs 2007, 68, 282-290.

18. Goldstein, E.D. Sacred moments: Implications on well-being and stress. J. Clin. Psychol. 2007, 63, 1001-1019.

19. Schmidt, M. Skala zur Erfassung von spirituellen Erfahrungen im Alltag: deutsche Adaptation mit Hilfe von Strukturgleichungsmodellen (Scale for the assessment of spiritual experiences in everyday life: German adaptation using structural equation models), Diplomarbeit, U of Frankfurt am Main, 2010.

20. Kalkstein, S.; Tower, R.B.; The Daily Spiritual Experience Scale and well-being: Demographic comparisons and scale validation with older Jewish adults and a diverse internet sample. J. Relig. Health 2009, 48, 401-417.

21. Zemore, S.E.; Kaskutas, L.A. Helping, spirituality and Alcoholics Anonymous in recovery. J. Stud. Alcohol 2004, 65, 383-391.

22. Stewart, C.; Koeske, G.F. A preliminary construct validation of the Multidimensional Measurement of Religiousness/Spirituality Instrument: A study of Southern USA. Int. J. Psychol. Relig. 2009, 16, 181-196.

23. Holder, M.D.; Coleman, B.; Wallace, J. M. Spirituality, religiousness, and happiness in children aged 8-12 years. J. Happiness Stud. 2008, 11, 131-150.

24. Pargament, K.I.; Smith, B.; Koenig, H.G.; Perez, L. Patterns of positive and negative religious coping with major life stressors. J. Sci. Study Relig. 1998, 37, 710-724.

25. Goggin, K.; Murray, T.; Malcarne, V.; Metcalf, K.; Gerkovich, M. What's God got to do with it? Adolescents' beliefs about God's role in their alcohol use. Presented at the 26th Annual Scientific Meeting of the Research Society on Alcoholism, Ft. Lauderdale, FL, USA, June 2003.

26. Oliveira, A.L.; Kimura, M.; Mishima, L.S. Cross-cultural adaptation and validation of the Daily Spiritual Experience Scale for the Brazilian culture. Qual. Life Res. 2010, 19 (Suppl. 1), 48.

27. Dean, M. Islam and psychosocial wellness in an American Afghan refugee community. PhD dissertation, Curtin University, Centre for International Health, 2006.

28. Underwood, L.G. The human experience of compassionate love: Conceptual mapping and data from selected studies. In Altruism and Altruistic Love: Science, Philosophy, and Religion in Dialogue; Post, S.G., Underwood, L.G., Schloss, J.P., Hurlbut, W.B., Eds.; Oxford University Press: New York, NY, USA, 2002; pp. 72-88.

29. Underwood, L.G. Chapter 1: Compassionate love: A framework for research. In The Science of Compassionate Love: Theory, Research and Applications; Fehr, B., Sprecher, S., Underwood, L.G., Eds.; Wiley-Blackwell: Malden, MA, USA, 2009; pp. 3-25.

30. Emmons, R.A.; McCullough, M. Counting blessings versus burdens: An experimental investigation of gratitude and subjective well-being in daily life. J. Personal. Soc. Psychol. 2003, 84, 377-389.

31. de Boer, A.G.; van Lanschot, J.J.; Stalmeier, P.F.; van Sandick, J.W.; Hulscher, J.B.; de Haes, J.C.; Sprangers, M.A. Is a single-item visual analogue scale as valid, reliable and responsive as multi-item scales in measuring quality of life? Qual. Life Res. 2004, 13, 311-320. 
32. General Social Survey Home Page. http://www.norc.uchicago.edu/GSS+Website/ (accessed on 15 December 2010).

33. Sanchez, E.G.M.; Arocena, F.A.L.; Ceballos, J.C.M. Daily spiritual experience in Basques and Mexicans: A quantitative study. J. Transpers. Res. 2010, 2, 10-25.

34. Loustalot, F.; Wyatt, S.B.; Sims, M.; Ellison, C.G.; Taylor, H.A.; Underwood, L.G. Psychometric testing of the Daily Spiritual Experiences Scale among African Americans in the Jackson Heart Study. J. Relig. Health 2009, doi: 10.1007/s10943-009-9278-2.

35. Duffy, R. Spirituality, religion, and work values. J. Psychol. Theol. 2010, 38, 1-17.

36. Maselko, J.; Kubzansky, L.D. Gender differences in religious practices, spiritual experiences and health: Results from the US General Social Survey. Soc. Sci. Med. 2006, 62, 2848-2860.

37. Koenig. H.G.; George, L.K.; Titus, P.; Meador, K.G. Religion, spirituality, and acute care hospitalization and long-term care by older patients. Arch. Intern. Med. 2004, 164, 1579-1585.

38. Koenig, H.G.; George, L.K.; Titus, P. Religion, spirituality and health in medically ill hospitalized older patients. J. Am. Geriatr. Soc. 2004, 52, 554-562.

39. Skarupski, K.; Fitchett, G.; Evans, D.; Mendes, C.F. Daily spiritual experiences in a biracial community-based population of older adults. Aging Men. Health 2010, 14, 779-789.

40. Boswell, G.H.; Kahana, E.; Dilworth-Anderson, P. Spirituality and healthy lifestyle behaviors: Stress counter-balancing effects on the well-being of older adults. J. Relig. Health 2006, 45, 587-602.

41. McCauley, J.; Tarpley, M.J.; Haaz, S.; Bartlett, S.J. Daily spiritual experiences of older adults with and without arthritis and the relationship to health outcomes. Arthritis Rheum. 2008, 59, 122-128.

42. McCracken, L.M.; Gauntlett-Gilbert, J.; Vowles, K.E. The role of mindfulness in a contextual cognitive-behavioral analysis of chronic pain-related suffering and disability. Pain 2007, 131, 63-69.

43. Rippentrop, A.E.; Altmaier, E.M.; Chen, J.J. The relationship between religion/spirituality and physical health, mental health, and pain in a chronic pain population. Pain 2005, 116, 311-321.

44. Campbell, J.D.; Yoon, D.P.; Johnstone, B. Determining relationships between physical health and spiritual experience, religious practices, and congregational support in a heterogeneous medical sample. J. Relig. Health 2010, 49, 3-17.

45. Park, C.L.; Brooks, M.; Sussman, J. Dimensiions of religion and spirituality in psychological adjustment in older adults living with congestive heart failure. In Faith and Well-being in Later Life: Linking Theory with Evidence in an Interdisciplinary Inquiry; Ai, A., Ardelt, M., Eds.; Nova Science Publishers: Hauppauge, NY, USA, 2009; pp. 41-58.

46. Ayo, H.T.; Agofure, S.H.; Adebukola, K. Psychosocial variables as predictors of work family conflict among secondary school teachers in Irele Local Government Area, Ondo State, Nigeria. Pak. J. Soc. Sci. 2009, 6, 11-18.

47. Woods, G. The 'bigger feeling': The importance of spiritual experience in educational leadership. Educ. Manag. Adm. Leadersh. 2007, 35, 135-155.

48. Holland, J.M.; Neimeyer, R.A. Reducing the risk of burnout in end-of-life care settings: The role of daily spiritual experiences and training. Palliat. Support. Care 2005, 3, 173-181.

49. Houston, D.J. Spirituality and public service. Public Adm. Rev. 2007, 16, 88-102. 
50. Stewart, C.; Koeske, G.F.; Koeske, R.D. Personal religiosity and spirituality associated with social work practitioners' use of religious-based intervention practices. J. Relig. Spiritual. Soc. Work: Soc. Thought 2006, 25, 69-85.

51. Voltmer, E.; Büssing, A.; Thomas, C.; Spahn, C. Religiosität, Spiritualität, Gesundheit und Berufsbezogene Verhaltensmuster bei Pastoren zweier freikirchlich-protestantischer Denominationen/Religiosity (Spirituality, health and work-related behavior patterns in pastors of two Free Protestant denominations). Psychother. Psych. Med. 2010, 60, 425-433.

52. Maisel, N.; Rauer, A.; Marshall, G.; Karney, B.R. Social support after a partner's traumatic injury: Situational, relationship, and individual difference predictors. In Support processes in intimate relationships; Sullivan, K., Davila, J., Eds.; Oxford University Press: New York, NY, USA, 2010; pp. 264-288.

53. Bell, D.E., Jr. The relationship between distal religious and proximal spiritual variables and selfreported marital happiness. PhD dissertation, Florida State University College of Medicine, 2009.

54. Brelsford, G.M.; Marinelli, S.; Ciarrochi, J.W.; Dy-Liacco, G.S. Generativity and spiritual disclosure in close relationships. Psychol. Relig. Spritual. 2009, 1, 150-161.

55. Fehr, B., Sprecher, S., Underwood, L.G. The Science of Compassionate Love: Theory, Research and Applications; Wiley-Blackwell: Malden, MA, USA, 2009.

56. Easterling, L.; Gamino, L.; Sewell, K.W.; Stirman, L.S. Spiritual experience, church attendance, and bereavement. J. Past. Care 2000, 54, 263-275.

57. Harris, S.K.; Sherritt, L.R.; Holdeer, D.W.; Kulig, J. Shrier, L.A.; Knight, J.R. Reliability and validity of the brief multidimensional measure of religiousness/spirituality among adolescents. $J$. Relig. Health 2008, 47, 438-457.

58. Van Dyke, C.J.; Glenwick, D.; Cecero, J.; Kim, S.K. The relationship of religious coping and spirituality to adjustment and psychological distress in urban early adolescents. Ment. Health Relig. Cult. 2009, 12, 369-383.

59. Desrosiers, A.; Miller, L. Relational spirituality and depression in adolescent girls. J. Clin. Psychol. 2007, 63, 1021-1037.

60. Park, C.L.; Edmondson, D.; Hale-Smith, A.; Blank, T.O. Religiousness/spirituality and health behaviors in younger adult cancer survivors: Does faith promote a healthier lifestyle? J. Behav. Med. 2009, 32, 582-591.

61. Shorkey, C.; Uebel, M.; Windsor, L.C. Measuring dimensions of spirituality in chemical dependence treatment and recovery: Research and practice. Int. J. Ment. Health Addict. 2008, 6, 286-305.

62. Allen, R.S.; Phillips, L.L.; Roff, L.L.; Cavanaugh, R.; Day, L. Religiousness/spirituality and mental health among older male inmates. Gerontol. 2008, 48, 692-697.

63. Ciarrocchi, J.W.; Deneke, E. Happiness and the varieties of religious experience: Religious support, practices, and spirituality as predictors of well-being. Res. Soc. Sci. Study Relig. 2005, 15, 209-233.

64. Ciarrocchi, J.W.; Deneke, E. Hope, optimism, pessimism, and spirituality as predictors of wellbeing controlling for personality. Res. Soc. Sci. Study Relig. 2005, 16, 161-183.

65. Roff, L.L.; Durkin, D.; Sun, F.; Klemmack, D.L. Widowhood, religiousness and self-assessed well-being among older adults. J. Relig. Spritual. Aging 2007, 19, 43-60. 
66. Dunn, K.S.; Wordlaw, S.L.; Chapleski, E. Everyday spirituality in central city elders. J. Relig. Spritual. Aging 2003, 15, 41-59.

67. Blumenthal, J.A.; Babyak, M.A.; Ironson, G.; Thorensen, C.; Powell, L.; Czajkowski, S.; Burg, M.; Keefe, F.J.; Steffen, P.; Catellier, D. Spirituality, religion, and clinical outcomes in patients recovering from an acute myocardial infarction. Psychosom. Med. 2007, 69, 501-508.

68. Mofidi, M.; DeVellis, R.F.; Blazer, D.G.; DeVellis, B.M.; Panter, A.T.; Jordan, J.M. Spirituality and depressive symptoms in a racially diverse US sample of community-dwelling adults. J. Nerv. Ment. Dis. 2006, 194, 975-977.

69. Watlington, C. The roles of religion and spirituality among African American survivors of domestic violence. J. Clin. Psychol. 2006, 62, 837-857.

70. Han, J.; Richardson, V.; The relationship between depression and loneliness among homebound older persons: Does spirituality moderate this relationship? J. Relig. Spiritual. Soc. Work: Soc. Thought 2010, 29, 218-236.

71. Bay, P.S.; Ivy, S.S.; Terry, C.L. The effect of spiritual retreat on nurses' spirituality: A randomized controlled study. Holist. Nurs. Pract. 2010, 24, 125-133.

72. Boelens, P.A.; Reeves, R.R.; Replogle, W.H.; Koenig, H.G. A randomized trial of the effect of prayer on depression and anxiety. Int. J. Psychiatry Med. 2009, 39, 377-392.

73. IN-CAM Outcomes Database Homepage. http://www.outcomesdatabase.org/ (accessed on 15 December 2010).

74. Miller, W.R.; Forcehimes, A.; O’Leary, M.J.; LaNoue, M.D.; Spiritual direction in addiction treatment: Two clinical trials. J. Subst. Abus. Treat. 2008, 35, 434-442.

75. Cole, B.S.; Hopkins, C.M.; Tisak, J.; Steel, J.L.; Carr, B.I. Assessing spiritual growth and spiritual decline following a diagnosis of cancer: Reliability and validity of the spiritual transformation scale. Psycho-Oncol. 2008, 121, 112-121.

76. Hall, D.E.; Koenig, H.G.; Meador, K.G. Episcopal measure of faith tradition: A context-specific approach to measuring religiousness. J. Rel. Health 2010, 49, 164-178.

77. Underwood, L.G. Interviews with Trappist monks as a contribution to research methodology in the investigation of compassionate love. J. Theory Soc. Behav. 2005, 35, 285-302.

(C) 2011 by the authors; licensee MDPI, Basel, Switzerland. This article is an open access article distributed under the terms and conditions of the Creative Commons Attribution license (http://creativecommons.org/licenses/by/3.0/). 